Rakenteiden Mekaniikka (Journal of Structural Mechanics)

Vol. 50, No 3, 2017, pp. $296-299$

https://rakenteidenmekaniikka.journal.fi/index

https://doi.org/10.23998/rm.64993

(c) The author(s) 2017.

Open access under CC BY-SA 4.0 license.

\title{
Magneto-structural simulation of an induction motor start-up using nodal magnetic forces in Elmer
}

\author{
Janne Keränen ${ }^{1}$, Pavel Ponomarev, Sabin Sathyan, Juhani Kataja and Anouar Belahcen
}

Summary. For reduction of vibrations and noise of electrical machines, the computation of the magnetic force distributions and the ensuing deformations are needed. Here we utilize nodal force method in open-source Finite Element software Elmer to compute the force distributions and the vibrations of an induction motor during start-up.

Key words: electromechanics, electromagnetics, structural mechanics, coupling, multi-physics, induction motor, vibrations

Received 19 June 201\%. Accepted 14 August 2017. Published online 21 August $201 \%$.

\section{Introduction}

Electrical machines are increasingly located close to the residential areas, offices, and industrial environment, where the noise of the devices is required to be rather low. For designing more silent electrical machines, the sources of the noise, e.g. the vibrations caused by magnetic fields, need to be analyzed accurately enough using numerical simulations. This paper concentrates on vibrations caused by the magnetic forces using so-called generalized nodal force method, and on magneto-structural coupling of Finite Element simulations.

For the numerical analysis of vibrations and noise of electrical machines, the computation of the accurate magnetic force distributions is a fundamental issue. There exists different electromagnetic force models - e.g. Maxwell stress tensor, virtual work principle, and different magnetization models. They are equivalent (up to numerical inaccuracies) in terms of the total net force acting on a magnetic body, but differ in force distributions and the ensuing deformations. Recently, one model based on virtual work principle, socalled Generalized Nodal Forces (GNF) have been researched [2, 5, 6]. The virtual work principle is logically stronger than the Maxwell stress tensor - one can derive the Maxwell stress tensor from it [1].

In this paper, we couple the generalized nodal forces (local mechanical loads obtained within electromagnetic simulation) to structural solver to solve the deformations due to the magnetic fields. We utilize open-source multi-physical FEM software Elmer from CSC-IT Center for Science, which excels in parallel computation [3].

${ }^{1}$ Corresponding author. janne.sami.keranen@vtt.fi 


\section{Generalized nodal forces and elasticity}

For generalized nodal forces, we utilize virtual work principle in one element of finite element mesh, take virtual displacement of nodes of the elements and differentiate the energy with respect to the displacement. The nodal force $\mathbf{F}$ can be obtained by differentiating the total magnetic energy subject to virtual displacements and transforming the magnetic flux densities in divergence preserving manner. Parametrizing the virtual displacements by a real number $s$ and requiring that the displacement map is identity at $s=0$, we obtain

$$
\mathbf{F}=-\lim _{s \rightarrow 0} \int_{\Omega}[\mathbf{H}(\mathbf{B})]^{T}\left[\frac{\partial J_{s}}{\partial s}\right] \mathbf{B}-\frac{\partial \operatorname{det} J_{s}}{\partial s}\left(\mathbf{H}(\mathbf{B}) \cdot \mathbf{B}-\int^{B} \mathbf{H}(\mathbf{B}) \cdot d \mathbf{B}\right) d \Omega,
$$

where $\mathbf{B}$ and $\mathbf{H}$ are the cartesian coordinate presentations of magnetic flux density and magnetic field strength, and $J_{s}$ is the Jacobian matrix of the virtual displacement $[2,5]$.

The deformations are solved from the dynamic equation of elastic deformation, i.e.

$$
\rho \frac{\partial^{2} d}{\partial^{2} t}-\nabla \cdot \tau=f
$$

where $d$ is the displacement, $\rho$ is the density, $f$ is the force density, and $\tau$ is the stress tensor. In anisotropic material, stress can be expressed by strain $\tau=E \epsilon$, where $E$ is the elasticity tensor. The linearized strain is $\epsilon=\frac{1}{2}\left(\nabla d+(\nabla d)^{T}\right)$. The nodal forces are utilized here as an excitation for structural analysis.

\section{Implementation in Elmer}

In this work, magnetostriction is omitted and the deformations are assumed to be so small that only one-directional coupling from electromagnetic solver to elastic solver is needed. Moreover, here we concentrate on 2D cases, even 3D would also work.

The electromagnetic subproblem can be solved with Elmer's MagnetoDynamics2D solver. The nodal forces are calculated as an intermediate post-processing step, where MagnetoDynamicsCalcFields solver computes the forces by keyword

Calculate Nodal Forces = Logical True

Further, a body force couples the nodal forces to a source field for the elasticity solver

Body Force 2

displacement 1 load dofs = equals "nodal force $1 "$

displacement 2 load dofs = equals "nodal force 2"

End

The StressSolver is utilized in those bodies, whose deformations need to be solved.

\section{Example case and results}

The example case is a $5 \mathrm{~kW}$ induction motor for blower applications. One of the authors have made an extensive tutorial about utilizing Elmer for solving the motor case in 2D and in $2 \frac{1}{2} \mathrm{D}$ (multi-slice model) [4]. The work here is an extension to this tutorial.

The motor is a three-phase two pole-pair induction motor with 48 stator slots, and 40 rotor slots. Details and dimensions can be found from the tutorial [4]. The tutorial explains step-by-step how to generate a 2D model for the motor. The electromagnetic model is driven by an electrical circuit, where sinusoidal voltages are given as input. In this paper, a version without utilization of symmetry is used.

The start-up of the induction machine example was simulated as a so-called direct online startup (DOL). There the rotation starts from zero speed and accelerates to the 
nominal speed, taking into account rotor inertia with simple kinematics solver [4]. Figure 1 presents the magnetic flux density in selected time-step in the steady-state mode in the end of the simulation.

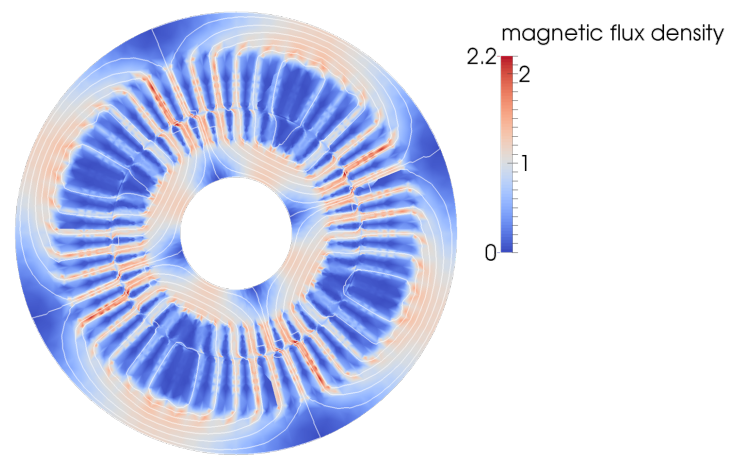

Figure 1. Magnetic flux density and flux lines in a selected time-step in steady-state mode.

The generalized nodal forces calculated from the solution of Figure 1 were used as source field for elasticity solver in Elmer. From the noise perspective, the most interesting domain for the elastic solver is the stator iron. Large magnetic forces acting on the stator core produce vibrations and the mechanical structure transmits and amplifies the vibrations, which result in the emission of noise. The forces in coils and insulators do not cause much vibrations, but they behave as additional mass, stiffness, and damping. However, the interface between the winding and the core is unclear and the material characteristics (e.g. stiffness) of coil-insulator combination region are rather vague. Hence, only the displacements of the stator core itself were solved here, and other stator parts were neglected. Further, for demonstrative purposes, also the deformations of the rotor iron were solved. As boundary condition, the tangential displacements on stator outer boundary and rotor inner boundary are set to zero. Figure 2 shows the rotor and stator displacements at first milliseconds after start-up and later in steady-state mode. The results show that the rotor is very stiff and the magnetic forces do not produce considerable deformation. In the beginning of the DOL start-up, the stator teeth displacements are considerably high.

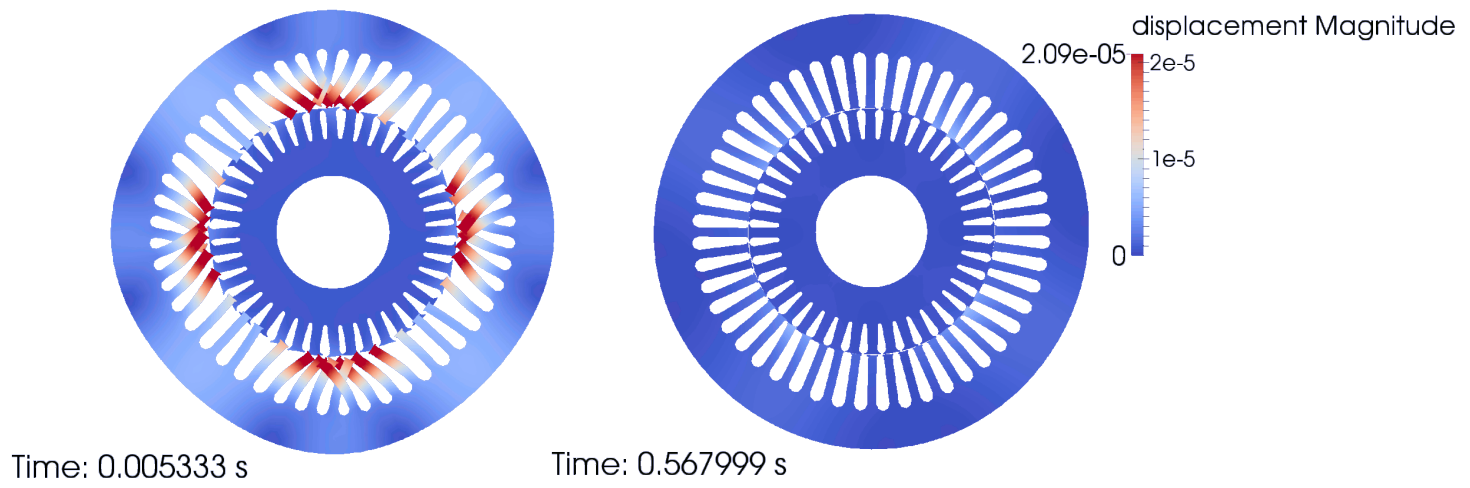

Figure 2. Displacement of the stator and rotor, the displacements are exaggerated by 500 times. Left: first milliseconds after the DOL start-up. Right: in steady-state phase.

Figure 3 shows the generalized nodal forces in the stator. The forces are concentrated at the teeth tips, but in the start-up phase there are considerable forces also in other surfaces of the teeth. In steady state, stator (and rotor) forces are mostly in radial direction, whereas in start-up there are also tangential components. The tangential components are bending the stator teeth during start-up, and stator yoke is deformed, too. In [5], similar computation results were validated to match with experimental results. 

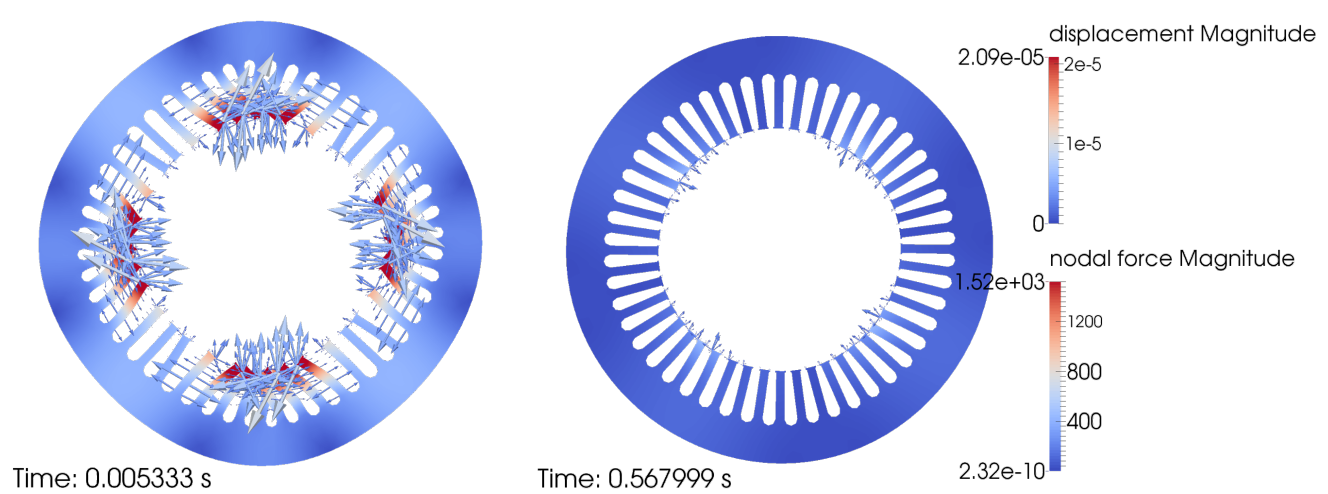

Figure 3. Nodal forces and displacements in stator, in start-up and steady state.

\section{Conclusion}

Computation of induction motor deformations based on magnetic forces has been demonstrated in Elmer. With the workflow of Elmer induction machine tutorial [4] and the extension shown in this paper, the same computation can be utilized in other cases, too.

\section{References}

[1] A. Bossavit. Virtual power principle and Maxwell's tensor: which comes first?. COMPELThe international journal for computation and mathematics in electrical and electronic engineering, 30(6):1804-1814, 2011. https://doi.org/10.1108/03321641111168110

[2] A. Kameari. Local force calculation in 3D FEM with edge elements. International journal of applied electromagnetics in materials, 3(1), 231-240, 1993.

[3] J. Keränen, J. Pippuri, M. Malinen, J. Ruokolainen, P. Råback, M. Lyly and K. Tammi. Efficient parallel 3-D computation of electrical machines with Elmer. IEEE Transactions on Magnetics, 51(3), 2015. https://doi.org/10.1109/TMAG.2014.2356256

[4] P. Ponomarev. Elmer FEM - Induction Machine Tutorial. VTT Report, 2017. https: //doi.org/10.13140/RG.2.2.18599.75688

[5] S. Sathyan, A. Belahcen, J. Kataja, T. Vaimann and J. Sobra. Computation of Stator Vibration of an Induction Motor using Nodal Magnetic Forces. 2016 XXII International Conference on Electrical Machines (ICEM), Lausanne, 2016, pp. 2198-2203. https://doi. org/10.1109/ICELMACH . 2016.7732827

[6] S. Sathyan, A. Belahcen, J. Kataja, F. Henrotte, A. Benabou and Y. Le Menach. Computation of Magnetic Forces Using Degenerated Air-Gap Element. IEEE Transactions on Magnetics, 53(6), 2017. https://doi.org/10.1109/TMAG.2017.2656239

Janne Keränen, Pavel Ponomarev

VTT Technical Research Centre of Finland Ltd

P.O. Box 1000, FI-02044 VTT, Finland

janne.sami.keranen@vtt.fi, pavel.v.ponomarev@gmail.com

Sabin Sathyan, Anouar Belahcen

Aalto University

Department of Electrical Engineering and Automation

P.O. Box 15500, FI-00076 Aalto, Finland

sabin.sathyan@aalto.fi, anouar.belahcen@aalto.fi

Juhani Kataja

CSC-IT Center for Science

P.O. Box 405, FI-02101 Espoo, Finland

juhani.kataja@csc.fi 